\title{
Quaderni
}

QUADERNI Communication, technologies, pouvoir

91 | Automne 2016

L'innovation dans tous ses états - II

\section{L'innovation, le nouvel imaginaire du changement}

Innovation : the new imaginary of change

\section{Danilo Martuccelli}

\section{(2) OpenEdition}

\section{Journals}

\section{Édition électronique}

URL : http://journals.openedition.org/quaderni/1007

DOI : 10.4000/quaderni.1007

ISSN : 2105-2956

\section{Éditeur}

Les éditions de la Maison des sciences de l'Homme

Édition imprimée

Date de publication : 5 octobre 2016

Pagination : 33-45

\section{Référence électronique}

Danilo Martuccelli, «L'innovation, le nouvel imaginaire du changement », Quaderni [En ligne], 91 | Automne 2016, mis en ligne le 05 octobre 2018, consulté le 02 janvier 2020. URL : http:// journals.openedition.org/quaderni/1007; DOI : 10.4000/quaderni.1007 


\section{$D$ ossier}

\section{L'innovation, \\ le nouvel \\ imaginaire \\ du changement}

\author{
Danilo \\ Martuccelli
}

Université Paris Descartes, USPC, membre de l'IUF CERLIS-CNRS
Si l'innovation a longtemps été une pièce à conviction - nullement la plus importante - du processus de modernisation, la crise des autres grands référents du changement, à commencer par l'idée du Progrès, lui octroie une centralité toute nouvelle dans l'imaginaire du changement. Nous étayerons cette hypothèse en trois grands moments. D'abord, nous reviendrons rapidement sur l'imaginaire moderne du changement et son lien avec l'idée du Progrès afin de souligner le rôle que, parmi d'autres facteurs, a alors eu la technique. Ensuite, nous montrerons comment la crise de cet imaginaire a permis d'accorder à l'innovation technique un surplus d'attention et d'efficacité symbolique. Enfin, nous présenterons quelques-uns des grands contours du nouvel imaginaire de l'innovation.

\section{Le Progrès et l'imaginaire moderne du changement}

Toutes les sociétés sont soumises, même à des rythmes différents, au changement. Mais à la différence des sociétés dites traditionnelles qui s'évertuaient à refouler le changement au nom de la conservation du passé (Balandier, 1974), les sociétés modernes ont fait du changement la clef de voûte de leur imaginaire. La notion d'imaginaire renvoie à la fois aux significations communes propres à un collectif social et à la capacité créatrice des sociétés et des hommes. (Leblanc, 1994). Autrement dit, l'imaginaire désigne à la fois un univers symbolique objectivé et un espace de capacités d'énonciation et de transformations symboliques. Le mouvement, qui jusqu'à l'avènement des temps modernes était considéré comme un signe d'imperfection, est devenu une valeur et l'horizon irréductible de la 
vie sociale. Plus ou moins en lien avec la Providence, une conscience spécifique du changement a fini par se forger : les hommes avaient la capacité de gouverner et de faire l'Histoire. Si ce processus se déroulait au milieu d'une situation donnée dont la genèse et la forme échappaient largement aux hommes, l'Histoire n'en était pas moins le résultat de leur dessein volontaire (Koselleck, 1990 ; Touraine, 1973).

Ce projet, et la croyance qui l'a accompagné, reposaient sur deux principes différents : d'une part, une compréhension volontariste de la réalité sociale comme œuvre humaine et d'autre part, une confiance dans un sens de l'Histoire - le Progrès - en tant que version à peine laïcisée d'une pensée métaphysique. Le projet de faire l'Histoire n'a été ainsi de mise, autant dans les discours intellectuels que dans ses reprises politiques, que lorsque ces deux principes sont allés de pair - quand le « sens » de la téléologie a informé le « mouvement » des faits.

Tout en étant l'objet d'une série de critiques (antimodernes ou contre-modernes), la confiance dans le Progrès a véritablement informé les regards que les individus portaient sur l'histoire et la modernité. Au fond, les hommes n'ont pas davantage fait l'Histoire dans la modernité que dans tout autre ; en revanche, ils ont conçu leur rapport à l'Histoire d'une manière spécifique. De la pauvreté aux inégalités scolaires, en passant par l'économie et la politique internationale : tout était scandé par la confiance dans le Progrès et dans les capacités plus ou moins illimitées d'intervention sur le monde. Les choses étaient injustes parce qu'elles apparaissaient comme inacceptables; et elles étaient inacceptables parce qu'il y avait la certitude qu'elles pouvaient être changées. L'Histoire et le changement étaient une affaire de volonté.

Cette confiance inusitée dans la volonté humaine s'est incarnée dans une série de grandes figures de la créativité humaine. À l'opposé des sociétés traditionnelles et de leur valorisation du passé et donc de l'imitation, les sociétés modernes ont construit autour de la création un tout nouveau credo civilisationnel (Rosenberg, 1962 ; Gomá Lanzón, 2014). La révolution, bien sûr et d'abord, cesse de signifier une restauration du passé pour annoncer l'avènement du radicalement nouveau ; un élan prolongé par l'avant-gardisme créateur des artistes modernes, la révolution industrielle et la destruction créatrice des capitaines d'industrie. Dans ce processus et surtout dans cette représentation générale de l'histoire, l'innovation n'est alors, au mieux, qu'une pièce à conviction supplémentaire : son sens et son rythme sont saisis à la lumière d'un essor plus général. C'est l'idée du Progrès qui fédère les différentes dimensions - de la politique à la morale, de l'art à la technique donnant aux hommes le sentiment qu'ils étaient capables de réaliser, ici et maintenant, sur terre, l'idéal que la religion chrétienne avait déposé dans l'au-delà (Strauss, 1992 ; Manent, 2012). L'avenir s'annonçait, en dépit des crimes et des catastrophes, radieux ; l'imaginaire du Progrès, en devenant hégémonique, répandra l'idée que toutes les couches sociales y avaient bien droit.

Sans négliger la force de la secousse produite par la thèse de l'héliocentrisme, puis par les Lumières, ce n'est que lorsque la science bâtit une nouvelle alliance avec la technique (dans la première et surtout la deuxième révolution 
industrielle) que la connaissance devient (est perçue et s'auto-perçoit) comme une source de libération des limites imposées jadis par l'ignorance et la base d'une promesse prométhéenne illimitée. Au cours de cette période, celle de la modernité conquérante, le projet prométhéen prime et enveloppe le projet technique à proprement parler. La nature particulière de la technique dans le temps moderne (une articulation entre la science et l'industrie) est ainsi indissociable d'un projet de maîtrise - la construction d'un monde différent capable de se superposer au monde naturel existant. C'est par ce truchement que la technique participe de l'imaginaire de puissance des modernes ; elle symbolise la volonté des hommes de s'affranchir des limites imposées par la nature. Le volontarisme atteint son apothéose. La nature, la physis, n'est plus le fondement de l'être ; sa place est occupée par un sujet qui devient le véritable fondement de la réalité, d'autant plus qu'il est capable de la plier à ses desseins, grâce à sa volonté (Heidegger, 1958 ; Ortega y Gasset, 1982).

Le poids de la technique dans ce processus ne peut donc pas, on le comprend aisément, être négligé ; mais elle n'avait alors de sens qu'à l'intérieur du culte voué au Progrès. Elle n'était qu'une expression, pour importante qu'elle se révélât, d'un projet illimité de pouvoir. Certes, la technique ne s'insère pas passivement dans ce qui est, selon Arendt (1994), le grand signe de la condition humaine dans les temps modernes - le primat indiscutable de la vita activa sur la vita contemplativa; elle donne forme à une vita activa où progressivement la praxis politique cède le pas à la poiesis technique, ce qui préfigure les problèmes que la généralisation d'un mode d'intervention sur la nature, dans ses excès prométhéens, produira au $\mathrm{XX}^{\mathrm{e}}$ siècle. Mais soulignons l'essentiel : ce n'est qu'à l'intérieur d'un imaginaire plus large et plus général du changement, que la science et la technique ont pris sens dans cette première étape.

\section{Crises et inflexions}

Cette conception du Progrès et cet imaginaire du changement sont entrés en crise depuis quelques décennies. Le constat en a été maintes fois effectué. Paul Ricœur en donne une vision synthétique. En tout premier lieu, l'illusion de l'origine, autant dire la grande césure entre la tradition et la modernité, s'estompe au fur et à mesure qu'on prend acte que «les discordances entre les rythmes temporels des diverses composantes d'un phénomène social global rendent bien difficiles de caractériser globalement une époque comme rupture et comme origine " (Ricœur, 1991 : 383). En deuxième lieu, ayant cessé de croire à la marche du progrès, il y a une prise de conscience d'une série de mutations et de crises qui ne s'insèrent plus dans un avenir déterminé. Enfin, les sociétés modernes cessent de croire à la fusion entre une théorie de l'Histoire et une théorie de l'action, autant dire au projet proprement prométhéen de faire l'Histoire.

À la lumière de ces crises, se répand un sentiment collectif d'impuissance d'un nouveau genre. Si l'avenir ne devient pas nécessairement sombre, en revanche, il se déleste d'un sens d'ensemble et de toute garantie eschatologique. Il est plus un avatar qu'un destin ; il est soumis à différentes contingences et à bien des risques. L'Histoire, toujours ouverte à la créativité humaine, ne tient 
plus à la seule volonté des hommes. Le rapport à l'Histoire se structure à partir d'une autre forme de conscience : les événements, sous l'effet d'interdépendances multiples et hiérarchisées, ne seraient plus sous contrôle.

Le brouillage du Progrès a une conséquence majeure au niveau de la perception du mouvement des événements et de l'expérience du changement. Sans horizon, la fragmentation du temps, l'urgence, l'accélération s'emparent des individus. La perte de sens de l'avenir donne forme à un tout nouveau sentiment d'accélération et de désorientation. Si dans le cadre de la société industrielle, la vie quotidienne a été l'objet de très importants changements qui ont profondément bouleversé, dès les premières décennies du XIX ${ }^{\mathrm{e}}$ siècle, la conscience des individus (Thompson, 1988), les manifestations ultérieures de ces changements (de l'électricité au téléphone, de la radio à la voiture, puis des soviets aux droits sociaux) pouvaient cependant être perçues à l'intérieur d'un cadre collectif d'intelligibilité - celui de l'idée de Progrès justement. Le mouvement et l'accélération d'événements, malgré leur importance, avaient un sens en référence à un avenir.

L'implosion de ce cadre change radicalement la donne historique. L'expérience de l'accélération, par exemple, se transforme (Rosa, 2010). Elle ne s'insère plus dans l'avènement d'un système technicien (Ellul, 1977), et elle ne fait plus, non plus, partie de l'imaginaire de la mobilité et du mouvement modernes (Barrère, Martuccelli, 2005); elle devient une expérience vertigineuse, aliénante et sans répit de changement au quotidien. Ce qu'on dénomme parfois le présentisme
(Hartog, 2003), n'est que l'expression au niveau de la conscience historique d'une réalité plus ordinaire et peut-être plus importante : la vie quotidienne, dans son accélération, s'autonomise du sens historique du Progrès. Résultat : la vie quotidienne est prise dans une urgence et une excitation qui font défaut aux événements historiques ; la vie personnelle, dans son intensité, semble aller plus « vite » que l'histoire collective. Le brouillage du Progrès alimente une expérience généralisée de mouvement et de vitesse au niveau de la vie personnelle qu'amplifie à son tour le brouillage de sens du Progrès. L'expérience de l'Histoire, comme des enquêtes empiriques le montrent, s'en trouve bouleversée, comme si nous assistions à une séquestration du mouvement par l'excitation tous azimuts du quotidien, faute de disposer d'un sens collectif et d'un espoir téléologique (Jedlowski, 2005 ; Martuccelli, 2006 ; Affuso, 2010). La profusion des événements et leur urgence au quotidien épaississent le présent, mais enlèvent aussi de l'épaisseur à chaque événement qui ne devient qu'un moment de passage, plus ou moins court, au sein d'un horizon dépourvu de direction.

Toutefois ce processus n'entraine pas seulement l'occlusion du futur dans le présent. La modernité conquérante n'a pas seulement été organisée autour du futur ; la distance entre l' « actuel » et le simple présent a été tout aussi significative. L'actuel : c'est-à-dire la présence, par anticipation incarnée, ici et maintenant, du futur. $\mathrm{Au}$ fond, c'est peut-être cela le véritable noyau de la crise. L'actuel n'a plus vraiment de valeur et surtout d'épaisseur face au présent. Prise en tenaille entre des événements historiques que la crise du progrès opacifie et une vie ordinaire qui 
s'accélère dans ses transformations, la conscience de l'Histoire vacille et derrière elle, l'imaginaire moderne du changement. Les individus font partout l'expérience du changement, mais ils sont de plus en plus incapables de le comprendre. Ils se sentent alors prisonniers d'une vie collective insensée et se méfient des avatars d'une Histoire dépourvue de sens.

Cette crise de l'idée du Progrès et de l'imaginaire du changement a connu une expression majeure du côté de la créativité humaine. Certes, malgré maints assauts critiques, l'agir créatif n'a pas vraiment été banni en tant que fondement de la modernité - comme en attestent d'ailleurs, dans les sciences sociales, les réflexions sur l'agency depuis trente ans (Giddens, 1987 ; Joas, 1999). Il n'empêche qu'une inflexion a bien eu lieu. La créativité cesse d'être le parangon des Grands auteurs ou inventeurs pour devenir une expérience plus ordinaire. Cette inflexion a parfois été interprétée à partir d'une sociologie des intellectuels. La transformation des récits sur la création serait l'expression d'un malaise des clercs, lié à leur perte de confiance dans l'ancien sujet historique - le prolétariat - ou à leur capacité à être les véritables législateurs du monde (Lyotard, 1979 ; Bauman, 1987). Mais ce malaise est aussi marqué par l'épuisement d'une certaine capacité de création artistique, qui amène l'époque à s'auto-représenter en termes de réélaboration et de répétition, l'originalité n'étant que la capacité à déplacer des signes dont l'origine est oubliée (ou refoulée). Une nouvelle période s'insinue, la postmodernité, qui « se caractérise non seulement comme nouveauté par rapport au moderne, mais plus radicalement comme dissolution de la catégorie de nouveau » (Vattimo, $1987: 10$ ).
Conséquence : la rupture cesse d'être la grande figure du changement. Cette transformation est tout aussi visible dans le domaine politique où la révolution a fini son cycle (Furet, 1995) que dans le domaine de l'art, avec l'avènement d'un paradigme contemporain en rupture avec la rupture du modernisme (Heinich, 2014). Progressivement, un nouvel imaginaire du changement, plus modeste, voit le jour : il s'organise autour d'une série de réformes, de mini-modifications, des usages et des tactiques, de valorisation de l'imitation, autour d'une série constante d'inflexions. Des successifs changements de cap à l'intérieur d'une certaine continuité, déplacent et prennent la place de l'ancienne figure de la grande rupture.

Or, la rupture avec l'imaginaire du changement comme rupture, si visible et même centrale dans la politique et dans l'art contemporain, l'est infiniment moins du côté de la technique. Résultat la technique devient plus que jamais l'horizon de l'utopie. Bien entendu, dans les temps modernes, la technique a depuis longtemps été porteuse des rêves d'immortalité, d'abondance et de puissance, et elle a aussi été, au moins depuis le succès de la notion d'aliénation, objet d'une série de critiques quant à ses dérives pratiques. La littérature - de Frankenstein aux catastrophes de la sciencefiction contemporaine - n'a cessé de souligner les dangers associés aux apprentis-sorciers (Jameson, 2008). Mais ces mises en garde ne prenaient leur véritable sens, comme on l'a évoqué, qu'à l'intérieur d'une vision prométhéenne plus large de l'Histoire. D'ailleurs chez ses critiques, l'ambivalence envers la technique a toujours été de mise : si d'un côté, on dénonçait l'imposition des diktats du système technicien (croissance autonome, automatisme, absence de finalité, 
accélération...) de l'autre côté, la technique était toujours susceptible, en dépit de sa cristallisation autonome en dehors de la volonté des hommes, de feedbacks positifs autant dans le domaine politique (grâce aux nouvelles possibilités de gestion et de légitimation qu'elle ouvre) que dans l'activité scientifique elle-même (la technique est un facteur incontournable du progrès) (Ellul, 1977 et 1988).

C'est en tenant compte de cet arrière-plan qu'il faut comprendre l'inflexion en cours. L'espoir et l'ambivalence dont la technique a été l'objet - dès les débuts du XIX ${ }^{e}$ siècle - lui permettent, aujourd'hui, à la lumière de la crise du Progrès, d'incarner, mieux que la politique ou que l'art, le nouvel imaginaire du changement. En tout cas, l'abandon ou l'épuisement de l'imaginaire de la création dans la politique ou l'art contrastent vivement avec ce qui est observable du côté de la science et de la technique. En fait, un mouvement vers le primat des technosciences comme clef de voûte du changement.

L'affirmation peut surprendre puisqu'à partir de la fin des années 1970, la conception de la science produite au XVII ${ }^{\mathrm{e}}$ siècle a été sérieusement ébranlée, ouvrant à une prise de conscience inquiète quant aux dégâts du progrès. À la différence de l'idéal positiviste du XIX ${ }^{\mathrm{e}}$ siècle, le savoir scientifique contemporain, en rompant avec des conceptions déterministes, s'est ouvert à des visions soulignant la complexité, la discontinuité, le hasard et le chaos. La potentialité de prédiction des sciences naturelles, encore fortement enracinée au niveau du sens commun, est de moins en moins partagée par bon nombre des scientifiques : la non-linéarité des phénomènes s'oppose à la linéarité, la complexité à la simplification, les problèmes introduits par la flèche du temps se généralisent, les conceptions d'une nature moins mécanique et plus active et créative, fondée sur l'auto-organisation, s'imposent.

D'un point de vue culturel et social, il revient à la postmodernité proprement dite et aux Sciences studies d'avoir livré un des grands diagnostics de cette transformation. Mouvements composites, ils ont théorisé l'abandon de l'idée de l'existence d'un possible langage unificateur de tous les discours et d'une science qui ne serait qu'un discours ventriloque neutre de la nature, au profit d'une conception soulignant la pluralité irrépressible des interprétations du monde. Même les preuves scientifiques sont désormais mieux reconnues dans leur irréductible composante de controverse et de désaccord. Or, c'est cela que la vérité scientifique avait justement promis dans le XVII ${ }^{e}$ siècle - en instituant la capacité de la Raison à s'ériger en tribunal du réel (Lyotard, 1979 ; Rorty, 1990). La mise en question de ce métadiscours transforme la connaissance, dépourvue désormais de fondements solides, en une série de « coups » pratiques réussis (Lyotard, 1983 ; Rorty, 1995 ; Pestre, 2013).

Puisque la réalité est l'objet de discours incommensurables, l'utilité pratique et l'opérationnalité des propositions deviennent les critères ultimes de la discrimination entre les représentations. Rien d'étonnant à ce que le principe de vérité du pragmatisme soit devenu depuis quelques décennies le nouveau critère hégémonique du vrai - que les auteurs s'en réclament ou non explicitement. Bien entendu, il serait absurde d'accorder à la postmodernité ou aux Sciences 
studies une audience qu'elles n'ont pas eue, mais elles ont cependant accompagné et vulgarisé l'idée d'une connaissance construite autour de controverses, de coups, de manipulations, l'idée donc d'un monde qui serait un champ agonistique de représentations. La raison n'aurait plus aucun accès privilégié à la réalité ou à la nature, et ne pourrait plus prétendre incarner un modèle universel et supra-historique. Chaque discours est contraint de générer par lui-même, et par la seule preuve de son efficacité, sa propre source d'autorité.

La profondeur de la crise de l'idée du Progrès, même si elle a des visages différents selon les domaines, n'en est pas moins le commun dénominateur de tous ces processus. L'imaginaire $\mathrm{du}$ changement et de la grande rupture, en tout cas, n'en sortent pas indemnes. Dans l'univers enchanté du Progrès, même la brutalité avait un sens. Lorsqu'on demanda à Stevenson, l'inventeur du train à vapeur, ce qui se passerait si une vache, dans l'Ouest américain, traversait les rails, sa réponse fut sans équivoque : tant pis pour la vache. La mobilisation, associée au progrès, pliait à l'avance toutes les objections. On n'arrêtait pas le Progrès.

Récapitulons : la politique et l'art sont marqués, l'une et l'autre, par l'épuisement d'un certain paradigme de la création; le sens métaphysique de l'Histoire s'érode ; la confiance dans la Raison humaine et les manifestations inexorablement positives du Progrès devient plus ambivalente. Ces différentes secousses n'ont pas aboli toute représentation positive du changement, notamment au niveau des représentations les plus ordinaires des individus, mais ont favorisé un déplacement significatif de l'imaginaire du changement en direction de l'innovation. Ce sera la dernière étape de notre argumentation.

\section{Le changement ordinaire ou l'imaginaire de l'innovation}

Peut-on vraiment faire de l'innovation la clef de voûte d'un nouvel imaginaire historique du changement ? Cette interprétation ne néglige-telle pas la force des discours critiques envers les méfaits de la technique ? N'est-il pas vrai que s'est répandue depuis presque 50 ans dans les sociétés occidentales, une conscience critique des méfaits du projet productiviste propre à la société industrielle?

L'intronisation progressive de l'imaginaire de l'innovation est à comprendre à l'encontre de ces objections et, pour cela, il faut partir des transformations évoquées dans le paragraphe précédent. D'ailleurs, la force même et la centralité des critiques envers la technique dans la période actuelle sont une pièce à conviction supplémentaire de la vigueur de ce nouvel imaginaire du changement (Beck, 2001). Si la technique concentre l'essentiel des critiques, c'est parce qu'elle est désormais censée être le principal lieu de fabrication du futur. Elle devient, plus que jamais, la réponse aux défis de l'avenir. Au-delà des critiques dont la notion a été la cible, le développement durable repose sur le pari du progrès technique : croissance verte ; véhicules à hydrogène, hybrides ou électriques ; nouvelle génération de réacteurs ; agriculture urbaine et extension de serres hydroponiques sur les toits des maisons ; bref, grâce à la technique, il serait possible de mettre en œuvre une économie moins consommatrice de 
ressources naturelles et moins dommageable pour l'environnement. Si la technique est à la base des défis écologiques posés par une société industrielle fondée sur le charbon, le pétrole et le gaz naturel, la technique est aussi à la base de l'espoir d'une société autour d'une économie fondée sur l'éolien, le solaire et la géothermie. La Green Economy sera la nouvelle fille prodigue, et même mûre, de l'idée du Progrès. Une attitude qui trouve une version extrême dans la croyance (si moderne, si naïve) quant à la fin de l'humanité et l'avènement de la post-humanité, les cyborgs, la Singularity ou la généralisation de l'automation via les robots (Besnier, 2012).

L'innovation est le mal et le bien. La croissance, par exemple, en réalisant ses promesses d'essor matériel, aurait donné lieu à une effroyable torsion du Progrès. La croissance exponentielle de la production industrielle est maintes fois dénoncée comme ingérable au vu des ressources limitées de la planète. Jusque-là considérée comme la grande réponse aux malheurs du monde, la croissance économique est soumise à la critique. Mais la plupart des critiques de la croissance, se font au nom d'un progrès dans lequel la technique, tôt ou tard, a un rôle majeur : que ce soit au niveau d'une meilleure utilisation des ressources naturelles ou dans le passage d'une société du plus à la civilisation du mieux. Si des critiques radicales existent, la critique porte davantage, dans la plupart des discours publics, sur un type de productivisme que véritablement sur une remise en question de l'imaginaire de la technique. Dans tous les cas, le contraste est flagrant avec l'enthousiasme productiviste et technique de Marx ou d'Engels, mais aussi avec l'expression célèbre de Schumpeter (1984: 116) pour qui le «processus de destruction créatrice constitue la donnée fondamentale du capitalisme».

Le sens et la direction des métaphores s'inversent. Hier, la révolution, d'origine politique, a fini par déteindre sur l'industrie ; aujourd'hui, l'innovation, de facture technique irradie vers le domaine politique et institutionnel. L'imaginaire du changement se calque sur les réalités de l'innovation, elles-mêmes privilégiant, comme foyer de prégnance métaphorique, l'innovation industrielle. L'importance accordée aux différentiels d'efficacité instrumentale, parfois au détriment même de l'ancienne notion de vérité scientifique, donne à la technique une nouvelle fonction épistémologique, politique et imaginaire. Elle donne forme à un imaginaire du changement que l'on peut représenter en analogie avec le mouvement d'un serpent avec des hauts et des bas.

D'une part, vers le haut, du côté de ce que la science avait jadis établi sur les bases d'un récit religieux millénaire, la technique serait aujourd'hui en train d'effacer le Grand Partage entre la nature et la culture, entre les humains et les non-humains, au fur et à mesure que de nouvelles entités sociotechniques et technoscientifiques apparaissent. La nature, qui pendant longtemps désignait un réel en dehors des capacités de création humaine, voit son statut se transformer profondément au fur et à mesure que la distance entre la science et la technologie se réduit et, avec elle, la frontière entre la nature, la culture et la société (Gibbons, 1994 ; Nowotny, Scott, Gibbons, 2001). Les technosciences - plus que la science - et infiniment plus que la politique ou l'art, deviennent le noyau de l'imaginaire du changement. Ce n'est pas un hasard si les dimen- 
sions les plus extrêmes de cette technophilie se manifestent aux États-Unis - du post-humain au projet Singularity, en passant par la sciencefiction - bien davantage qu'en Europe : après tout la troisième révolution industrielle est massivement un phénomène américain.

D'autre part, vers le bas, l'imaginaire de l'innovation est porteur d'une attitude infiniment moins modeste envers le changement au jour le jour. En fait, sournoisement, cet imaginaire prend des distances envers l'imaginaire du changement comme rupture. Cet état d'esprit s'étend dans des différentes domaines : l'époque des grandes découvertes pharmaceutiques seraient derrière nous; l'époque où la science-fiction prédisait des nouvelles techniques radicalement autres serait révolue (Graeber, 2015) au profit d'une innovation, plus ordinaire, infiniment moins spectaculaire. L'affirmation peut étonner tant elle semble aller à l'encontre de la révolution numérique et de la prolifération des innovations dont elle l'est l'objet. Pourtant, pour l'instant, comme le remarque de façon critique Graeber, la révolution informationnelle n'a pas vraiment donné lieu à des innovations objectales aussi révolutionnaires qu'ont pu l'être, jadis, les électrodomestiques ou l'impact que la vitesse a eu dans l'imaginaire de la modernisation dans les années 1960 (Ross, 2006). Mais au-delà de la pertinence - ou non - de cette critique, cela n'empêche pas l'innovation d'être porteuse d'un imaginaire ordinaire et permanent de changement, à la place d'une vision de la rupture et de l'extraordinaire, ce qui reflète d'ailleurs sa séduction au niveau des organisations (Crozier, 1995 ; Alter, 2000). L'innovation est une réalité constante, elle avance à petits-pas, elle vient «d'en haut » mais elle se prolonge " par le bas », grâce à la myriade des usages (alternatifs, customisations) des individus. L'abandon de l'imaginaire de la rupture est bien visible aussi, par exemple, dans les tendances à la dédifférenciation entre l'art culte et l'art populaire, une tendance activement prise en charge par la diversification tous azimuts des pratiques des acteurs (Glevarec, 2005).

Le visage de la troisième révolution industrielle est marqué avant tout par la vitesse des transformations des objets et des dispositifs (Rieffel, 2014) : ordinateurs, téléphones portables, smartphones, baladeurs, tablettes, streaming, e-book sans oublier YouTube, Facebook, Twitter, Gmail, Instagram, Google, Yahoo ou les blogs. Tous ces supports ont fini par transformer, en à peine quelques lustres, l'ensemble de la vie sociale, du travail à l'intime, de la consommation à la sociabilité ou à l'accès à l'information. Les produits se succèdent à une cadence fort rapide. Certains s'imposent plus ou moins dans la durée (comme la messagerie internet, même si désormais les individus consacrent plus de temps à leurs réseaux sociaux), d'autres comme les blogs, après un premier moment d'euphorie, décroissent sans pourtant disparaître tandis que d'autres encore deviennent plus ou moins obsolètes (comme le fax).

L'imaginaire de l'innovation permanente, tous azimuts, à tous les niveaux, en haut et en bas, donne forme à une nouvelle expérience du changement qui se dissocie de l'horizon du Progrès. Dans ce sens, l'imaginaire de l'innovation corrige l'affirmation, trop rapide, de ceux qui, à l'instar des postmodernes, ont annoncé le déplacement de la production vers la consommation en tant que secteur clé des sociétés actuelles (Lyon, 1994). 
L'innovation, au contraire, produit une nouvelle articulation entre ces domaines, l'un prolongeant l'autre, à tel point que les consommateurs deviennent sinon vraiment des producteurs (« prosumers ", amateurs), au moins des innovateurs par leurs usages (tactiques, braconnages, customisations). L'innovation donne un vocabulaire collectif aux expériences constantes, éphémères, ponctuelles, fragmentaires du changement, tout en soulignant leur caractère effréné et sans terme. La force de l'imaginaire de l'innovation c'est que, tout en se refusant à produire un récit ordonnateur des événements, il est toutefois capable de donner un sens au tourbillon du changement autrement que sous la figure du désordre ou du « bougisme » (Balandier, 1988 ; Taguieff, 2001). Le constat phénoménologique du changement incessant devient, grâce à l'imaginaire de l'innovation, une théorisation du social - parfois, la seule théorisation possible.

L'innovation - plus que la communication ou que la consommation - tend ainsi à devenir le centre de l'imaginaire du changement contemporain. À la différence du Progrès, ses grands méfaits et ses laissés pour comptes, l'innovation intègre et insère tous les individus - même de plus en plus rapidement au fur et à mesure que l'expansion des objets et des dispositifs techniques s'accélère et se généralise. À la différence de l'élitisme collectif ou individuel de l'avant-gardisme politique ou artistique, l'innovation - via ses usages et ses reformes ordinaires - alimente un imaginaire autrement plus égalitaire.

L'innovation, enfin, est en phase avec ce qui est une des grandes caractéristiques de l'expérience actuelle de la modernité et son rapport infini- ment plus circonspect, non seulement envers le Progrès, mais même avec le changement. Sur ce registre, nous sommes même en train de sortir de l'ambivalence propre à la modernité. Nous transitons de l'expérience d'une époque tiraillée sans point d'équilibre entre les méfaits et les bonheurs du changement, vers des expériences réclamant de plus en plus, au jour le jour, un droit généralisé d'inventaire. Il s'agit d'une des nouveautés majeures de la modernité au début du $\mathrm{XXI}^{\mathrm{e}}$ siècle. À la place de la foi dans le Progrès tend à s'imposer une autre attitude. La réflexion critique sur les innovations, ses usages et ses limites, irrigue désormais toute la vie sociale. Partout il est question d'évaluation et de bilans. En France, par exemple, étant donné l'importance des changements scientifiques dans ce domaine, les lois bioéthiques sont désormais révisées tous les cinq ans. Les thématiques ouvertes à controverse se multiplient dans une liste qui va de la GPA à la PMA, en passant par la recherche sur des cellules souches ou l'embryon, sans oublier les questions liées à la fin de vie. Devant toutes ces questions qui concernent la vie et l'humain, l'innovation technique n'est en elle-même porteuse d'aucune philosophie. Le contraste est saisissant avec la boutade de Stevenson à propos du train à vapeur. Face aux conséquences inquiétantes de l'innovation, même au milieu d'un âge séculier, la religion comme tradition morale redevient une source de réflexion critique majeure pour certains. À la lumière de cette transformation, les notions de mutation, sans parler de rupture ou de révolution ne semblent plus vraiment pertinentes pour cerner l'imaginaire contemporain du changement. Elles sont trop liées à la représentation d'un nouveau commencement et d'une créativité de l'agir radical. Pour décrire la situation et notre 
imaginaire actuel, la notion d'inflexion semble infiniment plus juste. Tout en signalant une modification de cap dans une trajectoire, la notion renvoie aussi à une forme de continuité avec le passé. Le changement se transforme. Il ne désigne ni une simple radicalisation des logiques déjà présentes, ni une véritable rupture avec les processus précédents. L'inflexion fait chair avec l'imaginaire de l'innovation : elle cerne le changement à la fois comme un nouveau stade et en profonde continuité, elle le décrit surtout comme une expérience ordinaire et pluridirectionnelle.

En conclusion de cet article, limitons-nous à présenter les trois grandes étapes de l'argumentation déployée :

1. L'idée du Progrès en tant qu'imaginaire du changement, sous la forte emprise d'éléments providentialistes, a établi un continuum de sens entre la politique et l'art, la science et la morale, dans lequel s'est insérée l'innovation ;

2. La crise de l'idée du Progrès a ouvert la voie à une série de discours critiques sur l'épuisement de la création ou sur le sens de l'Histoire, qui se sont traduits par une autonomisation du sens du changement dans les différents domaines, chacun devenant la cible de regards critiques particuliers - de la politique à l'art, en passant par la morale. Dans ce processus, la science mais surtout la technique commencent à prendre une importance croissante en tant qu'incarnations du changement ; elles résistent mieux au travail critique de sape du Progrès en partie parce qu'elles ont été naguère objet de discours plus ambivalents ;

3. À l'issue de ces transformations, la science et la technique, en fait, leur fusion progressive et la consolidation des technosciences font de l'innovation, ordinaire et permanente, le noyau dur du nouvel imaginaire du changement, en absence de toute image enchantée du Progrès et de l'Histoire. 


\section{$R \cdot E \cdot F \cdot E \cdot R \cdot E \cdot N \cdot C \cdot E \cdot S$}

O. AFFUSO, Il magazine della memoria, Roma, Carocci Editore, 2010.

N. ALTER, L'Innovation ordinaire, Paris, P.U.F. 2000.

H. ARENDT, Condition de l'homme moderne [1958], Paris, Presses Pocket, 1994.

G. BALANDIER, Anthropo-logiques, Paris, P.U.F., 1974.

G. BALANDIER, Le Désordre, Paris, Fayard, 1988.

A. BARRERE, D.MARTUCCELLI, « La modernité et l'imaginaire de la mobilité : l'inflexion contemporaine », in Cahiers internationaux de sociologie, vol. CXVIII, janvier-juin 2005, pp. 55-79.

Z. BAUMAN, Legislators and Interpreters, Cambridge, Polity Press, 1987.

U. BECK, La Société du risque [1986], Paris, Aubier, 2001.

J.M. BESNIER, Demain les posthumains [2009] Paris, Pluriel, 2012.

M. CROZIER, La Crise de l'intelligence, Paris, Seuil, 1995.

J. ELLUL, Le Système technicien, Paris, Calmann-Lévy, 1977

J. ELLUL, Le Bluff technologique, Paris, Hachette, 1988.

F. FURET, Le Passé d'une illusion, Paris, Calmann-Lévy/Robert Laffont, 1995.

M. GIBBONS, The New Production of Knowledge, London, Sage, 1994.

A. GIDDENS, Constitution de la Société [1984], Paris, P.U.F., 1987.

H. GLEVAREC, « La fin du modèle classique de la légitimité culturelle», in E. Maigret, E. Macé (éds.), Penser les médiacultures, Paris, Armand Colin, 2005, pp. 69-102.

J. GOMÁ LANZÓN, Imitación y experiencia, Madrid, Editorial Taurus, 2014.

D. GRAEBER, La Bureaucratie, Paris, Les Liens qui Libèrent, 2015.

F. HARTOG, Régimes d'historicité, Paris, Seuil, 2003.

M. HEIDEGGER, « La question de la technique » [1953], Essais et conférences, Paris, Gallimard, 1958, pp. 9-48.

N. HEINICH, Le Paradigme de l'art contemporain, Paris, Gallimard, 2014.

F. JAMESON, Penser avec la science-fiction [2005], Paris, Max Milo, 2008.

P. JEDLOWSKI, Un giorno dopo l'altro, Bologna, Il Mulino, 2005.

H. JOAS, La Créativité de l'agir [1992], Paris, Cerf, 1999.

R. KOSELLECK, Le futur passé [1979], Paris, École des Hautes Études en Sciences Sociales, 1990.

P. LEBLANC, « L'imaginaire social. Notes sur un concept flou ", in Cahiers internationaux de sociologie, vol.XCII, 1994, pp. 415-434.

D. LYON, Postmodernity, Buckingham, Open University Press, 1994.

J.-F. LYOTARD, La Condition postmoderne, Paris, Minuit, 1979.

J.-F. LYOTARD, Le Différend, Paris, Minuit, 1983.

P. MANENT, Les Métamorphoses de la Cité, Paris, Champs-Flammarion, 2012.

D. MARTUCCELLI, Forgé par l'épreuve, Paris, Armand Colin, 2006.

H. NOWOTNY, P. SCOTT, M. GIBBONS, ReThinking Science, Cambridge, Polity Press, 2001. J. ORTEGA Y GASSET, Meditaciones de la 
técnica y otros ensayos sobre ciencia y filosofía [1939], Madrid, Revista de Occidente/Alianza, 1982.

D. PESTRE, À contre-science, Paris, Seuil, 2013. P. RICOEUR, Temps et récit [1985], Paris, Seuil, 1991, t-3.

R. RIEFFEL, Révolution numérique, révolution culturelle, Paris, Gallimard, 2014.

R. RORTY, L'Homme spéculaire [1979], Paris, Seuil, 1990.

R. RORTY, L'Espoir au lieu du savoir, Paris, Albin Michel, 1995.

H. ROSA, Accélération [2005], La Découverte, 2010.

H. ROSENBERG, La Tradition du nouveau [1959], Paris, Minuit, 1962.

K. ROSS, Rouler plus vite, laver plus blanc [1995], Paris, Flammarion, 2006.

J. SCHUMPETER, Capitalisme, socialisme et démocratie [1942], Paris, Payot, 1984.

L. STRAUSS, Qu'est-ce que la philosophie politique? [1959], Paris, PUF, 1992.

P.-A. TAGUIEFF, Résister au «bougisme », Paris, Mille et une nuits, 2001.

E.P. THOMPSON, La Formation de la classe ouvrière anglaise [1963] Paris, Maison des Sciences de l'Homme, 1988.

A. TOURAINE, Production de la société, Paris, Seuil, 1973.

G. VATTIMO, La Fin de la modernité [1985], Paris, Seuil, 1987.

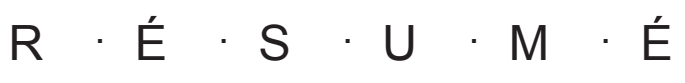

L'article propose une interprétation historique de l'émergence de l'innovation en tant que nouvel imaginaire du changement dans la période contemporaine. En s'appuyant sur des grandes représentations collectives, il étudie trois moments de ce déploiement. D'abord, il étudie la fonction somme toute limitée de l'innovation technique au cœur de l'idée du Progrès. Ensuite, il aborde la crise de cet imaginaire (notamment dans la politique et l'art) et le progressif surplus d'intérêt qui est accordé à l'innovation comme incarnation des transformations sociales. Enfin, il présente quelques-uns des grands contours de l'innovation en tant que nouvel imaginaire $d u$ changement.

\begin{abstract}
Innovation: the new imaginary of change

This paper develops an historical interpretation of the emergence of innovation as new imaginary of change in contemporary world. Departing from broad collective representations, it analyzes three moments of this process. Firstly, it addresses the limited function of technical innovation at the heart of the idea of Progress. Secondly, it studies the crisis of the imaginary of Progress in particular in politics and art and the increasing interest given to innovation as embodiment of social transformations. Finally, the paper presents and discusses some of the most salient features of innovation as new imaginary of change.
\end{abstract}


